\title{
Fuzzy Sensor Fusion and Curve Approximation for indoor Map Building
}

\author{
J.G. Juang, Y.C. Yang \\ Department of Communications \\ Navigation and Control Engineering National Taiwan Ocean University \\ Keelung, Taiwan
}

\begin{abstract}
This study uses a wheeled mobile robot to explore unknown indoor environment and build up a map of the unknown environment. The robot utilizes ultrasonic and infrared sensors with indoor positioning system to detect obstacles and identify unknown environment. Fuzzy system and sensor fusionare applied to control design. The proposed scheme can control the wheeled mobile robot move along walls and avoid obstacles. Angle and distance of the wheeled mobile robot are used to obtain the path trajectory. With sensed data of obstructions and walls, a map of unknown environment can be generated by curve fitting methods. The map building scheme is tested in different terrains and the experiments confirm that the proposed scheme works properly.
\end{abstract}

Keywords-sensor fusion; fuzzy system; mobile robot; map building

\section{INTRODUCTION}

In recent years, wheeled mobile robot (WMR) is one of the most discussed models in robotic researches. The WMR has been successfully applied to path planning, parking control, obstacle avoidance, navigation, object tracking, cooperation of multiple robots, etc. Kinematic model and stability analysis of the WMR are briefly reviewed as follows. Zhao [1] addressed six common kinds of three-wheeled robot model and their ability of control. Leow and his fellows analyzed the kinetic model of all-direction wheeled robot[2]. Chung[3] utilized two wheels different speeds to position control. Shi installed multiple sensors on WMR and utilized fuzzy expected value to control the WMR[4]. Lee[5] used a rapid path planning and fuzzy logic control to make the WMR trace an expected path. Fuzzy logic has the advantage that the solution to the problem can be cast in terms that human operators can understand, so that their experience can be used in the design of the controller. This makes it easier to merchandise works that are already successfully performed by humans and reduces the complexity of control system design. Fuzzy logic has the benefit of better promotion, greater fault tolerance and more fitting usage on nonlinear systems. In the development of the intelligent versatile automobile[6]-[8], the automobile used ultrasound sensors to help it search the correct reflective position. In actual environment, establish a route-map through the data that collected by the ultrasound sensor can help the automobile be practically and effectively. Most of other researches utilized visual sensors in map building. This study tries to simplify the control strategy, without using vision information, for a WMR in unknown environment exploration with less cost. Fuzzy theory and sensor fusion are applied to map building. As an intelligent system, the WMR integrates many functions such as environment sensing, dynamic decision-making and planning, behavior controlling and executing, etc. Since the WMR is a nonlinear model, dynamic equation is needed for system analysis and control regulation design. The fuzzy controller is one of the most prevalent controllers for nonlinear systems because the control scheme of fuzzy controller is simple and flexible. The distance obtained from the ultrasonic sensor is used as the input of the fuzzy controller. An infraredbased localization system called Star Gazzeris applied to the control scheme that provides the coordinate location to the WMR. Therefore, the trajectory path of the WMR obtained from the localization system is used as the reference. Without visual sensor the proposed sensor fusion system can still perform map building successfully.

\section{SYSTEM DESCRIPTION}

A WMR called Ihomer is used in the entity test. The control scheme mainly uses infrared sensor and ultrasound sensor to detect unknown environment around the WMR. The encoder of the WMR can serve as a feedback for the WMR. An infrared-based localization system offers the coordinates of the WMR. Additionally, the ultrasonic sensor is used to provide obstacle avoidance information. The encoders supply the measured value of the body movement, which are located at the side of the wheels. Ultrasound and infrared receivers measure the distance of objects and help collect the information that is used to build the map and to perform wall following. The WMR is located on the Cartesian coordinate system with no lateral or sliding movement as shown in Figure1. The movement distance of the WMR on global coordinate is

$$
\left(\begin{array}{c}
\dot{x} \\
\dot{y} \\
\dot{\theta}
\end{array}\right)=g_{1}(q) v+g_{2}(q) w=\left(\begin{array}{c}
\cos \theta \\
\sin \theta \\
0
\end{array}\right) v+\left(\begin{array}{l}
0 \\
0 \\
1
\end{array}\right)
$$

If $\Delta t<<1,(1)$ can be replaced by (2)

$$
\left[\begin{array}{l}
x_{k}(i+1) \\
y_{k}(i+1) \\
\theta(i+1)
\end{array}\right]=\left[\begin{array}{c}
x_{k}(i) \\
y_{k}(i) \\
\theta(i)
\end{array}\right]+\left[\begin{array}{cc}
\cos \theta & 0 \\
\sin \theta & 0 \\
0 & 1
\end{array}\right]\left[\begin{array}{l}
v \\
\omega
\end{array}\right] \Delta \mathrm{t}
$$




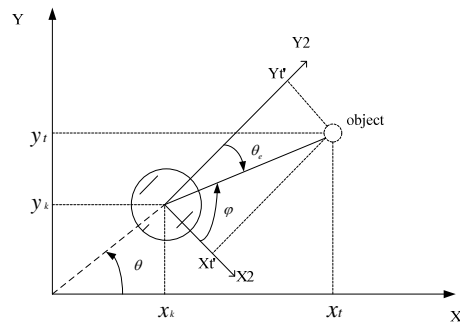

FIGURE I.COORDINATE OF RELATIVE POSITION OF WMR AND EXPECTED POSITION.

where $\mathrm{v}$ is the speed of the center of the body, $\mathrm{w}$ isthe angular speed of the center of the body,ris the radius of the wheel, $\mathrm{d}$ is distance between the left wheel and the right wheel, $\theta$ isthe included angle between the center of the body and $\mathrm{x}$ axis of the global coordinate system, $\theta$ eisthe relative angle of the heading direction of the WMR and expected position (error angle), ${ }^{x_{k}(i)}$ isx-axis coordinate of the center of the body on global coordinate system, $y_{k}(i)$ isy-axis coordinate of the center of the body on global coordinate system, $\Delta t$ issample time of every movement of the WMR, ${ }^{w_{l}}$ is left-wheel speed of the WMR, ${ }^{w_{r}}$ isright-wheel speed of the WMR.In order to obtain the relative distance, we change the target position and the WMR position from global coordinate system to the local coordinate, which is between the center of the WMR and the expected position.

$$
\left[\begin{array}{l}
x_{t}^{\prime} \\
y_{t}^{\prime}
\end{array}\right]=\left[\begin{array}{cc}
\sin \theta & -\cos \theta \\
\cos \theta & \sin \theta
\end{array}\right]\left[\begin{array}{l}
x_{t}-x_{k} \\
y_{t}-y_{k}
\end{array}\right]
$$

The WMR center relative distance and expected position (error distance) is

$$
d_{e}=\sqrt{x_{t}^{\prime 2}+y_{t}^{\prime 2}}
$$

The relative angle is defined as the included angle between the heading direction of the WMR and expected position. The local coordinates of the WMR obtained by (3) through the trigonometric function can be calculated.

$$
\varphi=\tan ^{-1}\left(\frac{y_{t}^{\prime}}{x_{t}^{\prime}}\right)
$$

The error angle of the heading direction and expected position is calculated by (6), as shown in Figure 1.

$$
\theta_{e}=90-\varphi
$$

where $\mathrm{X} 2$ is $\mathrm{x}$-axis of body coordinate system, $\mathrm{Y} 2$ isy-axis of body coordinate system, Xtisthe $\mathrm{x}$-axis coordinate of the expected position on the global coordinate system, Ytisthe $y$ axis coordinate of the expected position on the global coordinate system, $\mathrm{X} t$ 'isthe $\mathrm{X}$-axis coordinate of the expected position on the body coordinate system, Yt'isthe $y$-axis coordinate of the expected position on the body coordinate system, $\varphi$ isthe included angle between the lateral direction of the WMR and the expected position, deisthe relative distance between the WMR and expected position (error distance).

\section{CONTROL SCHEME}

In this paper, we propose a fuzzy control scheme that can drive the WMR to explore unknown environment. We put focus on how to keep the WMR move parallel to the wall at a constant speed. According to this way of moving, the mobile robot will automatically stop when it reaches the origin of the coordinates, then the environmental exploration is completed. Distance and angle information of the moving robot can be recorded, thus the position of the wall can be obtainedas shown in Figure2.

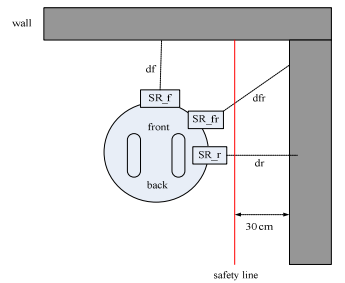

FIGURE II.SENSORS AND INPUT VARIABLES OF THE WMR.

There are two common inputs of the proposed fuzzy controllers for speed and angle control, which are the detected distances dfr and dr. These data are used to keep the WMR move on the track of safety line. The input variable for distance $\mathrm{dr}$ is SR $\mathrm{r}$ and the fuzzy values are very near, near, medium, far, and very far. The input variable for distance dfr is SR_fr and the fuzzy values are very near, near, medium, far, and very far. The output variable for turning angle is TA and the fuzzy values are TR3, TR2, TR1, TZ, TL1, TL2, TL3, which represent angle turn right very large, turn right large, turn right, go forward, turn left, turn left large, and turn left very large. Fuzzy speed is selected from distance factor. The fuzzy rules for turning angle are shown in Table 1. The speed of the WMR is fixed.According to current position of the WMR, heading angle, and the detected distance $\mathrm{dr}$, a point of the terrain at that location can be calculated. Path trajectory is formed by a series of locations, curves and straight lines are then obtained by the use of the first or second order curve fitting method. In this study, different approaches are applied to map building.

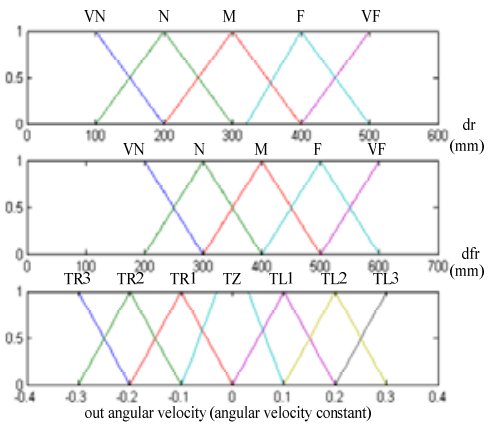

FIGURE III.FUZZY MEMBERSHIP FUNCTIONS OF THE INPUT AND OUTPUT VARIABLES. 
TABLE I.THE FUZZY LOGIC RULE TABLE.

\begin{tabular}{|l|l|l|l|l|l|}
\hline $\begin{array}{l}\text { SR_fr } \\
\text { SR__ }\end{array}$ & VN & $\mathbf{N}$ & $\mathbf{M}$ & $\mathbf{F}$ & $\mathbf{V F}$ \\
\hline VN & TL3 & TL1 & TZ & TZ & TZ \\
\hline N & TL2 & TR1 & TZ & TZ & TZ \\
\hline M & TL2 & TL1 & TZ & TZ & TZ \\
\hline F & TL2 & TL1 & TZ & TR1 & TR1 \\
\hline VF & TL2 & TL1 & TZ & TR2 & TR3 \\
\hline
\end{tabular}

\section{A. Least Squares Regression}

A curve or line with the minimal deviation from the data points can be derived by the method of least squares. The best fit curve would have the least square error for deviations from the data points to the line itself. In this method of least squares, the overall solution minimizes the sum of the squares obtained from the errors that are made while solving each equation. This gives the maximum likelihood of a variable assuming which the errors have a normal distribution.

$$
\begin{gathered}
b_{0}=\bar{y}-b_{1} \bar{x} \\
b_{1}=\frac{\sum\left(x_{i}-\bar{x}\right)\left(y_{i}-\bar{y}\right)}{\sum\left(x_{i}-\bar{x}\right)^{2}} \\
\hat{y}=b_{0}+b_{1} x_{i}
\end{gathered}
$$

Wherex is $\mathrm{x}$-axis coordinate, $\mathrm{y}$ is $\mathrm{y}$-axis coordinate, $\bar{x}$ is the average of $\mathrm{x}, \bar{y}$ is the average of $\mathrm{y}, \hat{y}$ is a linear equation.

\section{B. Linear Interpolation Method}

The method set up a new sampling point before and after the original sampling point to two adjacent connections, the connection through the new location of the sampling time when the value is set to the value of the new sampling point. If the two known points are given by the coordinates $\left(x_{0}, y_{0}\right)$ and $\left(x_{1}, y_{1}\right)$, the linear interpolation is the straight line between these points. For a value $\mathrm{x}$ in the interval $O$, the value y along the straight line is given from (10)

$$
\frac{y-y_{0}}{x-x_{0}}=\frac{y_{1}-y_{0}}{x_{1}-x_{0}}
$$

\section{Median Filter}

The main design uses a fixed-size mask. The median is calculated by first sorting all the data values from the surrounding neighborhood into numerical order and then replacing the data being considered with the middle data value[9]. The mathematical equations can be expressed as

$$
\begin{aligned}
m_{i j} & =\operatorname{median}(X) \\
X & =W_{i j}(I)
\end{aligned}
$$

wherem is the middle data valueof the surrounding neighborhoodXand $\mathrm{W}$ is the selected mask that is used to eliminate noise.

\section{Weighted Average Filter}

The output of a smoothing, linear spatial filter is simply the average of the data contained in the neighborhood of the filter mask[10]. The mask yields a so called weighted average, terminology used to indicate that data are multiplied by different coefficients, thus giving more importance to some data at the expense of others. In the mask shown in Figure 4 the data at the center of the mask is multiplied by a higher value than any other, thus giving this data more importance in the calculation of the average. The other data are inversely weighted as a function of their distance from the center of the mask. The diagonal terms are further away from the center than the orthogonal neighbors and, thus, are weighted less than the center point the highest data.

$$
\frac{1}{16} \times \begin{array}{|c|c|c|}
\hline 1 & 2 & 1 \\
\hline 2 & 4 & 2 \\
\hline 1 & 2 & 1 \\
\hline
\end{array} \quad X=\frac{1}{16} \sum_{i=1}^{9} z_{i}
$$

FIGURE IV.WEIGHTED AVERAGE FILTER MASK.

\section{EXPERIMENT RESULTS}

The experiments are tested in different terrains. One of the tests is shown in Figure 5.The right bottom of the figure is the origin of the coordinate, safe distance is $300 \mathrm{~mm}$.By the use of median filter approach the estimated map can be transferred to the blue line, as shown in Figure 6. Comparison of error of the coordinates by different approaches is shown in Table 2, where the percentage error is obtained from the average distance error divided by the total distance of the path.Maximal total average error of X-coordinate is $6.3 \mathrm{~cm}$ and Y-coordinate is $2.7 \mathrm{~cm}$. The median filter has best performance in map building.

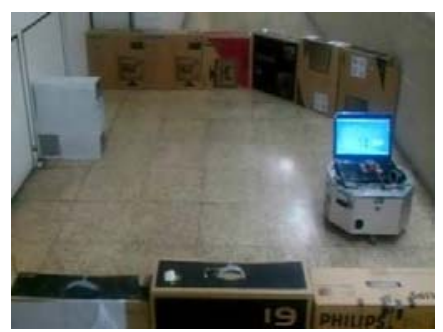

FIGURE V.TOPOGRAPHIC MAP OF THE ORIGINAL ENVIRONMENT.

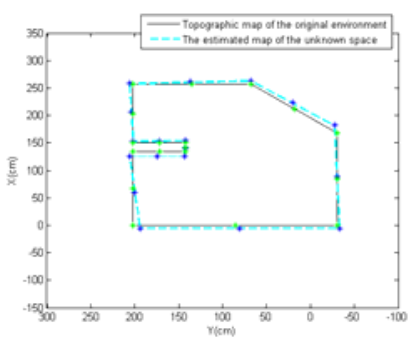

FIGURE VI.THE ESTIMATED MAP BY THE MEDIAN FILTER APPROACH. 
TABLE II COMPARISON OF COORDINATEERROR.

\begin{tabular}{|l|l|l|l|}
\hline \multirow{2}{*}{ Approaches } & \multicolumn{2}{|l|}{$\begin{array}{l}\text { Total average error } \\
\text { (cm) }\end{array}$} & $\begin{array}{l}\text { Percentage } \\
\text { error (\%) }\end{array}$ \\
\hline \multirow{2}{*}{ Leastsquares } & X-coordinate & 6.2 & 0.71 \\
\cline { 2 - 4 } & Y-coordinate & 2.1 & 0.24 \\
\hline \multirow{2}{*}{$\begin{array}{l}\text { Linear } \\
\text { interpolation }\end{array}$} & X-coordinate & 6.3 & 0.72 \\
\cline { 2 - 4 } $\begin{array}{l}\text { Weighted } \\
\text { average }\end{array}$ & Y-coordinate & 2.6 & 0.30 \\
\cline { 2 - 4 } Medianfilter & Y-coordinate & 5.9 & 0.68 \\
\cline { 2 - 4 } & X-coordinate & 2.7 & 0.31 \\
\cline { 2 - 4 } & Y-coordinate & 5.9 & 0.68 \\
\hline
\end{tabular}

\section{CONCLUSIONS}

In this paper, an intelligent map building scheme based on fuzzy system and sensor fusion is proposed to control a mobile robot for building unknown environment map. This research integrates an infrared localization system and ultrasonic sensors to mark obstacles. According to ultrasonic sensor's location and the detected distance between the sensor and object, map building can be completed by curve fitting method after the environmental exploration is finished. The task of building map for unknown environment can be accomplished without using visual sensors. Accuracy of the map building is demonstrated through experimental results. Real-time PC based control of the WMR on wall-following and map building is performed successfully.

\section{REFERENCES}

[1] Y. Zhao, S.L. BeMent.Kinematics, Dynamics and Control of Wheeled Mobile Robots.Proceedings of the 1992 IEEE International Conference on Robotics \&Automation, pp. 91-96, 1992.

[2] Y.P. Leow, K.H. Low, W.K. Loh. Kinematic Modelling and Analysis of Mobile Robots with Omni-Directional Wheels. Proceedings of the Seventh International Conference on Control, Automation, Robotics And Vision, pp. 820-825, 2002.

[3] Y. Chung, C. Park, F. Harashima.A Position Control Differential Drive Wheeled Mobile Robot.IEEE Transactions on Industrial Electronics, 48(4),pp. 853-863, 2001.

[4] E.X. Shi, W.M. Huang, Y.Z. Ling.Fuzzy Predictive Control of Wheeled Mobile Robot Based on Multi-Sensors.Proceedings of the Third International Conference on Machine Learning and Cybernetics, pp. 439-443, 2004.

[5] T.H. Lee, H.K. Lam, F.H.F. Leung, P.K.S. Tam. A Practical Fuzzy Logic Controller for The Path Tracking of Wheeled Mobile Robots.Application Note IEEE Control Systems Magazine, pp. 60-65, 2003.

[6] P. Bai, H. Qiao, A. Wan, Y. Liu. Person-Tracking with Occlusion Using Appearance Filters.Proceedings of the IEEE/RSJ International Conference on Intelligent Robots and Systems,pp. 1805-1810, 2006.

[7] T. Darrell, G. Gordon, M. Harville, J. Woodfill. Integrated Person Tracking Using Stereo, Color, and Pattern Detection.Proceedingsof Conference on Computer Vision and Pattern Recognition, pp. 601-609, 1998.

[8] J.G. Juang, C.H. Wu. Type-2 Fuzzy Control of a Mobile Robot for Avoiding Moving Object.Key Engineering Materials, 474, pp. 13001305, 2011.

[9] N.C. Gallagher, G.L. Wise. A Theoretical Analysis of the Properties of Median Filters. IEEE Transactions on Acoustics, Speech, and Signal Processing, 29(6), pp. 1136-1141, 1981.

[10] Linear Interpolation,http://faculty.ksu.edu.sa/11843/interpolation/Linearinterpolation 12-14-06.pdf 